\section{Misunderstandings about developmental dyslexia: a historical overview}

\author{
Pietro Smirni, ${ }^{1}$ Luigi Vetri, 2 \\ Eliana Misuraca, ${ }^{3}$ Marco Cappadonna, ${ }^{4}$ \\ Francesca Felicia Operto, 5 Grazia Maria \\ Giovanna Pastorino, 5,6 Rosa Marotta ${ }^{7}$
}

1Department of Educational Sciences,

University of Catania, Catania;

2Departiment of Health Promotion,

Maternal and Child Health, Internal

Medicine and Specialist of Excellence

"G. D'Alessandro", University of

Palermo; ${ }^{3}$ Geriatric Unit, Department of

Internal Medicine and Geriatrics,

University of Palermo; ${ }^{4}$ Departiment of

Clinical and Experimental Medicine,

University of Messina; ${ }^{5} \mathrm{Child}$ and

Adolescent Neuropsychiatry Unit,

Department of Medicine, Surgery and

Dentistry, University of Salerno; 6 Sleep

Lab for Developmental Age, Clinic of

Child and Adolescent Neuropsychiatry,

Department of Mental Health, Physical

and Preventive Medicine, University of

Campania "Luigi Vanvitelli”, Napoli;

7Department of Medical and Surgical

Science, University "Magna Graecia",

Catanzaro, Italy

\section{Abstract}

Developmental dyslexia is a reading disorder unrelated to intellectual disability, inadequate teaching systems or poor motivation for schooling. The first attempts to understand such difficulty of learning to read, connected the problem to a primary 'visual defect'. Since then, several models have been developed. In the last decades, autopsy and histopathological studies on the brain of developmental dyslexics provided neuroanatomical evidence of structural and morphological differences between the normal and dyslexic brains. Furthermore, neuroimaging studies allowed to understand the neural systems of reading and dyslexia. According to more recent studies, developmental dyslexia appears as a language-related neurodevelopmental disorder with a deficit in phonological decoding and visuospatial organization of the language code. Therefore, dyslexia is viewed as a multicomponential and complex disorder. Consequently, rehabilitation should be aimed at both the recovery of linguistic decoding processes and the improvement of visuo-spatial and attentional processes. This brief overview should be a valuable tool for a deeper understanding of dyslexic disorder. Literature searches in Medline, PsycINFO, EMBASE, Scopus, PubMed, Web of Science identified one hundred articles focusing attention on how this disorder has been considered over the years.

\section{Introduction}

Misunderstandings about dyslexia have fairly distant historical roots, and although several clinical and research studies have contributed, over the years, to understanding dyslexia, still today there are many outstanding questions and developmental dyslexia, in clinical practice, continues to be an overused diagnosis. ${ }^{1,2}$

However, research and studies agree on what developmental dyslexia is not. ${ }^{3}$ It is not the difficulty to learn reading associated to intellectual disability, or to sensory deficits (visual, auditory) that impair the grapheme-phoneme conversion, or to education and cultural deprivation, or to psychopathological conditions that can modulate the relational, psychological or emotional problems of the child. 4,5 Each of these clinical, psychological or social conditions can give rise to learning reading difficulties. Nevertheless, these conditions are generally associated with wider learning or cognitive disabilities. A child with poor cognitive resources may show difficulties or delays in learning reading due to abstraction difficulty in decoding alphabetical abstract symbols. This learning disability cannot be considered as dyslexia. It is rather a poor general intelligence that will obviously affect reading ability. ${ }^{6}$. Likewise, sensory deficit, cultural or educational deprivation, psychological difficulties can also cause problems in learning to read, which, however, cannot be considered as dyslexia. $2,7,8$

However, the long historical journey of clinical and experimental research allowed the researchers to reach some certainties: 1 . Reading disorders may occur despite a good intelligence, a good verbal comprehension and production, the lack of visual or auditory disorders, sufficient motivation and environmental opportunities to learn. ${ }^{2} 2$. Dyslexic subjects are able to correctly perceive both real objects and their visual representations. 1,93 . However, they find difficult to correctly read the written names of the same objects and can revert, omit, substitute written letters or words, showing difficulty in decoding the symbolic written language system, regardless their visual system or general intelligence.3,9 4. Visual defects 'per se' do not seem to explain the origin of a specific learning difficulty to read. 10,11
Correspondence: Luigi Vetri, Del Vespro 129, 90127 Palermo, Italy.

Tel.: +39 3286434126

E-mail: luigi.vetri@gmail.com

Key words: Developmental dyslexia, phonological decoding, attention.

Contributions: substantial contributions to the design of the study: PS, LV, MC; references search: EM, LV, MC, GMGP; drafting the paper: PS, FFO, MC, EM, RM; Revising the paper: PS, LV, FFO, EM, MC, GMGP, RM.All the authors read and approved the final manuscript.

Conflict of interest: The authors declare no potential conflict of interest.

Funding: None.

Availability of data and materials: All data and materials are included in the main text.

Ethics approval and consent to participate: Not required

Informed consent: Not required.

Received for publication: 16 February 2020

Revision received: 18 April 2020.

Accepted for publication: 14 May 2020.

This work is licensed under a Creative Commons Attribution NonCommercial 4.0 License (CC BY-NC 4.0).

(C) Copyright: the Author(s), 2020

Licensee PAGEPress, Italy

Pediatric Reports 2020; 12:8505

doi:10.4081/pr.2020.8505

\section{Materials and Methods}

The aim of the current study was to collect an overview of the references reporting a brief history of reading disorder, referred to as developmental dyslexia, over the years. The practical implication may be to better understand such reading disorder and improve the quality of treatment programs for dyslexic subjects.

One hundred articles were analysed, including case report, review and metaanalysis. Pre-1990 articles have been excluded from the analysis, except for some to describe the historical excursus of the theoretical models on developmental dyslexia. Further exclusion criteria were the studies that did not add historical interest to the topic. A total of fifty-seven articles have been included in the current review using as search criteria keywords such as: neurodevelopmental disorders, reading disorder, 
developmental dyslexia, attention deficit, phonological decoding, reading, dyslexia history. Studies were identified through electronic database searching in Medline (Ovid, 1946 to present), PsycINFO (Ovid, 1806 to present), EMBASE (Ovid), and adapted for Scopus (Elsevier), ERIC (Proquest), PubMed and Web of Science (ISI). The final database search was run on January 2020.

\section{From a 'visual defect' to a multi- component disorder}

Over a hundred years ago, assuming that an intelligent teenager had to learn to read, a British school physician could not understand why a smart and intelligent young boy, 14-year-old, had learning reading problems. ${ }^{11}$ Therefore, the school doctor diagnosed his young patient's reading disorder as a visual defect suggesting a sort of therapeutic 'eyes training'.12

Similarly, a few years later in 1917 , Hinshelwood, an Edinburgh ophthalmologist, considered dyslexia as a form of 'congenital blindness for words' and carefully distinguished the reading disorder from other clinical conditions in which total or partial inability to read may occur, such as poor intelligence, sensory difficulties, specific hearing impairment. Moreover, he hypothesized that the 'purity of symptoms' should be the crucial feature of dyslexia and, as for acquired blindness for words in adults, he postulated that the biological basis of the disorder was agenesis or developmental defect of the left angular gyrus. ${ }^{13}$

In the first decade of the twentieth century, it was accepted as undoubted that dyslexia was for childhood the analogue of the 'acquired blindness for the words' of the adult. In contrast, on the basis of reading and spelling mistakes of dislexics, Orton viewed dyslexia as a failure in establishing cerebral dominance and accounted it as a language disorder. ${ }^{14,15}$ However, as the difficulty of learning to read has become more widely recognized as such, inconsistent data have emerged on its neurological basis, its nature and causes as well as on its existence as a clinical entity. 15

In the past, some authors denied dyslexia as a specific clinical entity ascribing it to factors such as teaching methods, inadequate family influences, emotional factors and primary visual defects. 16 Then, two different frameworks emerged. The first considered the visuo-perceptual defect as the core of dyslexia and the other associated it with social and relational factors, such as poor education or emotional and psychological problems. ${ }^{17,18}$ Moreover, the distinction between developmental dyslexia and other categories of reading defects disappeared. As a consequence, a large body of studies collected conflicting data. These conceptual divergences persisted for a long time until the diagnosis of dyslexia was considered absurd and even a 'mystique of learning difficulties' was hypothesized. ${ }^{19}$ According to some authors most of children classified as dyslexic would rather be the result of inappropriate reading teaching methods. ${ }^{20}$ These extreme assumptions raise the problem of the diagnostic criteria to be used. ${ }^{21}$ The criteria were often different among the various researchers who considered subjects of different clinical populations as belonging to a single group of dyslexics. The most widespread criterion proved to be grossly inadequate: the child who has a reading level of two or more years lower compared to the attended class is dyslexic. ${ }^{21}$ This criterion does not take into account the intellectual level, the class (the first grade or the third grade), the cultural background of the quality of the school. The simple two-years criterion leads to overdiagnosis, while more rigid criteria risk false negatives, diagnosing as nondyslexic subjects who are instead dyslexic. ${ }^{21}$ However, even when restrictive criteria were used, dyslexics differed from one another in terms of neurological, behavioral or psychometric performance. Therefore, different classifications were elaborated 1 . A first one differentiated a 'pure dyslexia' from a 'dyslexia plus', depending on whether it was accompanied by 'soft' neurological signs. ${ }^{22}$ 2. A second one, considering the different patterns of reading errors, identifies three different dyslexic groups: the dysphonetic group, unable to integrate symbols with their sounds, the dyseidetic group, unable to perceive letters and whole words as configurations, and a mixed group with a combination of both dysphonetic and dyseidetic deficiencies. $^{23} 3$. A third classification considers the cognitive profile of dyslexics highlighted by the performances in verbal and non-verbal tests. 24,25 It therefore seemed increasingly evident that dyslexia could be a multicomponent disorder, rather than a disorder resulting from a single visuo-spatial, visuo-motor or phonological problem. Therefore, the roots of developmental dyslexia move from the eyes to the brain. In the following years, a large number of researches has shown that the phenomenology of dyslexia manifests itself in a multifaceted modality with difficulties both in the visuo-spatial and in the phonological processing. ${ }^{26}$

\section{Neural basis of reading and dyslexia}

Data from brain imaging studies in

\section{Evidence from autopsy and neu- roimaging studies}

Around the second half of the last century, research on dyslexia reached a new impetus from the histo-pathological and neuroimaging studies. ${ }^{27-29}$ For the first time, autopsy studies on the brain of developmental dyslexics showed a bilaterally large temporal planum, and the absence of the planum temporal asymmetry. ${ }^{30}$ In addition, such studies found, in the whole brain, cytoarchitectonic abnormalities, such as ectopic collections of neurons in layer one of the cortex, especially in perisylvian areas, in the thalamus lateral geniculate nucleus of the visual pathway and in the medial geniculate nucleus of the auditory pathway. Such cortical anomalies were more frequent in the areas surrounding Silvio's fissures, in the left hemisphere, roughly corresponding to the frontal and temporal language areas.

Both reduced planum temporal asymmetry and cortical malformations suggest an anomalous brain development, in prenatal stages of corticogenesis, with abnormally high levels of surviving neurons, as a significant neuroanatomical association to developmental dyslexia. ${ }^{31}$

Such abnormal neural migration affects the pattern of connectivity within and between the hemispheres, especially with the thalamus, ipsilateral and contralateral cortex and alters the development of these brain areas. Neuron ectopic collections may be associated with a developmental anomaly of the adjacent cortical layers (dysplasia).

Along with dysplasia and cortical ectopias, vascular anomalies often resemble small micro angiomas and sometimes arterio-venous malformations. Neuroradiological studies also showed a link between dyslexia and arteriovenous malformations, especially in males and in the left superior temporal areas.32,33

Post-mortem and neuro-imaging studies provided strong neuroanatomical evidence on the structural and morphologic differences between normal and dyslexic brains, giving a cogent contribution to understanding the neural and cognitive basis of developmental dyslexia as a language-based disorder. 34 
developmental dyslexia documented interrelated neural reading systems both in posterior and in anterior brain regions. 35

The cerebral posterior system includes two different and related structures: 1. a parieto-temporal area activates in analysis of the single word units (phonemes) and includes parts of the supramarginal gyrus in the inferior parietal lobule, parts of the posterior aspect of the superior temporal gyrus and parts of the angular gyrus in the parietal lobe. 2. an occipito-temporal area, referred as the visual word-form area, activates for skilled and fluent reading. ${ }^{36}$

The anterior system, instead, encompasses the left inferior frontal gyrus (Broca's area) and the dorsal and ventral premotor regions and is associated with articulation and silent reading and naming. 37,38

In dyslexic readers the anterior reading system, in the left hemisphere, appeared slightly overactivated while the two posterior systems were under activated, strongly supporting the "neural signature of dyslexia'. 35

This neuroanatomical consistent evidence allows to understand the neural basis of reading and dyslexia as a language-based disorder supported by a basic deficit in phonological processing. ${ }^{35}$

\section{Phonological model}

In recent decades, on the basis of neurobiological and neuroimaging studies, a new model of dyslexia has emphasized the role of language-phonological processing. ${ }^{39}$ According to such a model, the core of dyslexia is a selective, specific impairment of the language-processing at the phonological level.

Learning to read implies the ability to associate spoken sounds with written letters and to reach a phonological awareness according to which written words represent the sounds of spoken words. ${ }^{2}$ The word 'man' derives by the phonemes $\mathrm{m} / \mathrm{a} / \mathrm{n} / \mathrm{as}$ individual sounds combined with each other. Such word can be identified, understood and stored as the word 'man' after being analyzed in its individual phonemic units. In the spoken language, a genetic phonological module processes individual phonemes combining with each other into the word.

However, spoken language is a 'natural' system and the 'combination' of the phonemes is 'automatic'. Written language, instead, is a 'conventional' system and reading is much more demanding and requiring a more intentional activity. The written word must be phonologically decoded and identified and, subsequently, understood by higher-level cognitive functions, such as intelligence and vocabulary. 2,7,39

Therefore, reading implies a consciously awareness of the phonological form of words. Such phonologic awareness may be lacking in dyslexic readers. Therefore, a phonological deficit in the ability to segment the written word into its underlying elements, impacts on the functionality of the higher-level linguistic processes involved in the attribution of meaning, in learning or recalling complex, new, long words, or in mechanical memorization and rapid 'naming'.

Thus, two different but integrated neurocognitive processes are involved in reading: the first decodes the written symbolic linguistic code and the second one decodes the visuo-spatial characteristics of the same linguistic code. An impairment in one of the two neurocognitive processes or in both results in the reading disorder referred to as developmental dyslexia. However, written language is firstly a symbol system, although it is expressed in visuo-spatial graphemes, shapes, dimensions. A single clustering of letters configures different meanings according to the spatial relation between individual letters (w/a/s is "was" and s/a/w is "saw"). Conversely, a capital or small letter has the same value, as a linguistic symbolic code, regardless of its visuo-spatial features. Letters such $\mathrm{p} / \mathrm{b} / \mathrm{d} / \mathrm{q} / \mathrm{n} / \mathrm{u}$ are codes whose linguistic value is closely associated with their visuo-spatial features and orientation (right/left/up/down). The organization of a visual stimulus results from the 'meaning' assigned to the stimulus. In reading, 'meaning' of the visual stimuli is linguistic in nature and the lacking integration between letters and words derives from the inability to associate linguistic (the meaning) and visual dimension of the written word. ${ }^{9}$ In addition, reversal and perceptual errors are limited to printing in their own language and are therefore linguistic rather than visual. ${ }^{9,27}$

A deficit in the phonological elaboration of written language tends to slow down or compromise performance in all tasks requiring word phonological segmentation and analysis, in the decoding of complex written texts and in the processes of reading automatization, in the timed reading and comprehension of the text, in the processes of new episodic verbal learning and in the mechanical memorization of verbal material (tables, dates, nomenclatures, lists of words not associated), in the rapid naming and recalling of words, in the decoding and in the functional use of words without con- text, in the use of complex, long or unfamiliar and abstract words, in the answers to multiple choice written questions, in reading and writing of foreign words. ${ }^{40,41}$

Many 'compensated' adult dyslexics may learn to read and can even excel in their academic performance and gain access to higher levels of the language system with excellent achievements. ${ }^{42}$ However, this often requires high costs and resources. Moreover, it can be compromised in particular contexts and in stressful situations, such as, for example, a condition of written examination or a written timed task which puts a strain on the ability to quickly make decisions on phonological data. Some timed tests reveal that decoding remains very laborious for dyslexics: their ability to identify words is not automatic and it is characterized by extreme slowness in making decisions based on phonological data. ${ }^{2}$

\section{Compensated dyslexia and read- ing strategy}

Analysing a written text of an exam of an Italian thirty year old compensated dyslexic, graduated in law, we highlighted relevant morphological errors concerning the name declination, the verb conjugation, using the functors (articles, prepositions, conjunctions) and the phonological distortions, omissions and substitutions (e.g. 'la Supra Corte' for 'la Suprema Corte' 'originarimente' for 'originariamente'; 'maggiormente dibatti' for 'maggiormente dibattuti'; 'ad un contratto agenzia' for 'ad un contratto di agenzia'; 'l'assenza di qualsiasi rapporto impediscono' for 'l'assenza di qualsiasi rapporto impedisce'; 'l'esercizio non consentono' for 'l'esercizio non consente'; 'dei mezzi del reato' for 'dei mezzi di esecuzione del reato'; 'i coniugi provvedeva' for 'i coniugi provvedevano').

Interestingly, errors were higher in the fair copy of the text than in the rough draft. 'La Suprema corte', in the fair copy, for example, is 'la Supra Corte', (with the obvious omission of the syllable 'ma'), while in the rough draft was properly written. The quality of errors and their frequency can be considered as residual traces of a compensated dyslexic disorder. Moreover, the greater frequency of errors in the fair copy may be associated with the prevalent use of a semantic strategy. The good reader encodes words in a flexible way either by an analytical phonological approach to individual graphemes or by a global semantic approach to the whole words, more related to the meaning of the word in the context. The familiar word 'mother' may be correct- 
ly read with a low phonological analysis and its meaning may be semantically perceived from the context. Conversely, a less familiar, longer, or more complex word, such as 'electroencephalogram', requires a phonological analysis of the various graphemes. In a semantic approach, the word 'walked' can be read as 'walks' if, in context, 'walks' appears as a significant meaning. The dyslexic reader favors semantic strategy, strongly associated with the meaning of words and context, rather than phonological strategy associated with the analysis of individual graphemes.

On these assumptions, in the dyslexic lawyer, during a written examination, the fair copy (that is the second version) probably represents a 'more automated' transcription of an already known and repeatedly read text, that no longer requires a phonological analysis. In this way, morphological errors may more easily appear. Conversely, in the rough draft (that is the first version), the words still represent a new production and they may be written with a more analytic phonological analysis and adherence to their orthographic structure. Therefore, morphological errors in the compensated dyslexic lawyer should be re-interpreted as a phonological impairment and as a preferential use of semantic strategy that focuses more on the meaning than the phonological analysis of the text.

\section{Reading and cognitive processes}

For the planning of targeted interventions and individual recovery programs, the identification of the cognitive abilities underlying the dyslexic reading disorder is crucial. In addition to phonological processing, numerous studies have focused on the links between phonological recoding and other cognitive processes, in particular on attention mechanisms. ${ }^{29}$ The generation of phonological codes from written words is not an automatic process but demands attentional resources, particularly in the development of fluent and skilled reading. Rather, attention is a critical and overlooked cognitive dimension, for translating print into speech and achieving fluent and automatic reading. ${ }^{43}$ Therefore, attention may be a crucial factor in reading and in reading difficulties. For example, attention-deficit/hyperactivity disorder and dyslexia are frequently observed in the same individual. 44

Moreover, since the 1960s, several studies have highlighted the presence of naming-speed deficit as a second core deficit in dyslexia. 45 Further research revealed orthographic factors, extending the double-deficit hypothesis (phonological deficits and naming-speed deficits) to the triple-deficit hypothesis.46,47 According to such hypothesis, visual processing of orthographic information is viewed as an independent factor contributing to word recognition. 48 Researchers have also suggested further deficits in poor reading. There is a body of evidence supporting that dyslexic readers could be deficient in basic visual processes. 49,50

\section{Genetical basis}

The relationship between genetic information and dyslexia continue to be a highly complex topic. Many family aggregation studies and, even more, twin studies are compatible with a genetic etiology for dyslexia. ${ }^{51}$

Moreover, several studies have highlighted a strong genetic basis and an high familiarity in the pathogenesis of dyslexia. Six candidate genes have been identified and there is evidence that four of these are involved in a genetic system crucial for axon growth and neuronal migration, in the prenatal brain development, 27,52 affecting the timing of early neural migration and normal connectivity patterns in the brain. 51

Chromosomal regions have been identified where the presence of dyslexia would appear to be linked to the susceptibility of some genes, ${ }^{53}$ suggesting that a disturbance in the neuronal migration and a reduced activity in the left hemispheric brain regions are pathophysiological correlates of dyslexia.

It seems that genetic influences appear to be most relevant on severe dyslexia, while mild or soft dyslexia may be less influenced by genes than environment. 51 Therefore, it will probably need to identify the forms of family dyslexia that are most associated with genetic factors and to develop more targeted interventions for people at risk from the early school years.

\section{Conclusions and practical impli- cations}

Therefore, in recent decades, advances in genetic, neurobiological and psychological research have provided a significant contribution for understanding of a reading learning disorder difficult to interpret. 54,55 However, many questions about the best definition and treatment of dyslexia are still outstanding.

As a practical implication, knowledge of the history of developmental dyslexia should allow an approach to the problems of dyslexic subjects based on research acquisitions and on the neuronal basis of this developmental selective language-related disorder.

This brief historical overview of developmental dyslexia studies, from the early reports suggesting a visual defect or focusing on the reversal errors, showed that developmental dyslexia should be considered as a heterogenous neurodevelopmental language-based disorder in learning to read, in decoding letters/sounds, in accuracy and word recognition, primarily resulting from both phonological processing and visuo-spatial and visuo-attentional impairment. $29,44,56,57$ Therefore, rehabilitation must focus on the recovery of language and visuo-spatial and visuo-attentional processes underlying the complex and multi-component developmental dyslexia. 29

Moreover, a large body of studies showed that dyslexia does not compromise per se reasoning skills, problem solving ability, conceptualization, critical thinking and vocabulary. 2,3,7,35 Thus, the compensated dyslexics can use the 'general framework' of theories, models, and ideas. However, when long lists of unfamiliar words have to be memorized or when details are not linked by associated ideas or theoretical frameworks, dyslexics can be really disadvantaged and can show difficulties with mechanical memorization, rapid retrieval of words and repetition of words on request. 2

Moreover, while a good reader decodes words using phonological or semantic strategy, dyslexics often favor a semantic strategy and refer to the context to identify individual words. This strategy slows down their reading and compromises a valid phonological analysis.

\section{References}

1. Richardson SO. Historical perspectives on dyslexia. J Learn Disabil 1992;25:40-7.

2. Shaywitz SE. Dyslexia. N Engl J Med 1998;338:307-12.

3. Lyon GR, Shaywitz SE, Shaywitz BA. A definition of dyslexia. Ann Dyslexia 2003;53:1-14.

4. Operto FF, Precenzano F, Bitetti I, et al. Emotional Intelligence in Children with Severe Sleep-Related Breathing 
Disorders. Behav Neurol 2019

5. Smirni D, Precenzano F, Magliulo RM, et al. Inhibition, set-shifting and working memory in Global Developmental Delay preschool children. Life Span Disabil 2018;21:191-206.

6. Smirni D, Smirni P, Di Martino G, Operto FF, Carotenuto M. Emotional Awareness and Cognitive Performance in Borderline Intellectual Functioning Young Adolescents. J Nerv Ment Dis 2019;207:365-70.

7. Shaywitz SE, Shaywitz BA. Dyslexia (specific reading disability). Biol Psychiatry 2005;57:1301-9.

8. Smirni D, Smirni P, Carotenuto M, et al. Noli Me Tangere: Social Touch, Tactile Defensiveness, and Communication in Neurodevelopmental Disorders. Brain Sci 2019;9:368.

9. Vellutino FR. The validity of perceptual deficit explanations of reading disability: A reply to Fletcher and Satz. J Learn Disabil 1979;12:160-7.

10. Facoetti A, Paganoni P, Turatto M, et al. Visual-spatial attention in developmental dyslexia. Cortex 2000;36:109-23.

11. Morgan WP. A case of congenital word blindness. Br Med J 1896;2:1378.

12. Stein J. The magnocellular theory of developmental dyslexia. In: Reading and Dyslexia. Springer; 2018. p. 10334.

13. Hinshelwood J. Congenital word-blindness. HK Lewis \& Company, Limited; 1917.

14. Orton ST. Reading, writing and speech problems in children. 1937;

15. Geschwind N. Why Orton was right. Ann Dyslexia 1982;13-30.

16. Gates AI. The psychology of reading and spelling with special reference to disability. Teachers college, Columbia university; 1922.

17. Carotenuto M, Esposito M, Cortese S, et al. Children with developmental dyslexia showed greater sleep disturbances than controls, including problems initiating and maintaining sleep. Acta Paediatr 2016;105:1079-82.

18. Carotenuto M, Messina A, Monda V, et al. Maternal stress and coping strategies in Developmental Dyslexia: an italian Multicenter study. Front Psychiatry 2017;8:295.

19. Coles G. The learning mystique: A critical look at" learning disabilities". Pantheon Books; 1987.

20. Zigmond N. A prototype of comprehensive services for secondary students with learning disabilities. Learn Disabil Q 1978;1:39-49.

21. Benton A. Exploring the history of neuropsychology: Selected papers. OUP
USA; 2000.

22. Denckla MB. Minimal brain dysfunction and dyslexia: Beyond diagnosis by exclusion. Top child Neurol 1977;24361.

23. Boder E. Developmental dyslexia: A new diagnostic approach based on the identification of three subtypes. J Sch Health 1970;40:289-90.

24. Mattis S. Dyslexia syndromes: A working hypothesis that works. Dyslexia An Apprais Curr Knowl 1978;43-58.

25. Watson BU, Goldgar DE. Evaluation of a typology of reading disability. J Clin Exp Neuropsychol 1988;10:432-50.

26. Menghini D, Finzi A, Benassi M, et al. Different underlying neurocognitive deficits in developmental dyslexia: a comparative study. Neuropsychologia 2010;48:863-72.

27. Peterson RL, Pennington BF. Developmental dyslexia. Annu Rev Clin Psychol 2015;11:283-307.

28. Boets B, Wouters J, Van Wieringen A, Ghesquiere P. Auditory processing, speech perception and phonological ability in pre-school children at highrisk for dyslexia: A longitudinal study of the auditory temporal processing theory. Neuropsychologia 2007;45:160820.

29. Peters JL, De Losa L, Bavin EL, Crewther SG. Efficacy of dynamic visuo-attentional interventions for reading in dyslexic and neurotypical children: A systematic review. Neurosci Biobehav Rev 2019;100:58-76.

30. Galaburda AM. Developmental dyslexia: A review of biological interactions. Ann Dyslexia 1985;35:19-33.

31. Galaburda AM. Ordinary and extraordinary brain development: Anatomical variation in developmental dyslexia. Ann Dyslexia 1989;39:65-80.

32. Kemper TL. Asymmetrical lesions in dyslexia. Cereb Domin Biol Found 1984;75-89.

33. Wajuihian SO. Neurobiology of developmental dyslexia: Part 1: A review of evidence from autopsy and structural neuro-imaging studies. African Vis Eye Heal 2011;70:199-202.

34. Shaywitz SE, Shaywitz BA, Pugh KR, et al. Functional disruption in the organization of the brain for reading in dyslexia. Proc Natl Acad Sci 1998;95:2636-41.

35. Shaywitz SE, Shaywitz BA. Paying attention to reading: the neurobiology of reading and dyslexia. Dev Psychopathol 2008;20:1329-49.

36. Cohen L, Dehaene S, Naccache L, et al. The visual word form area: spatial and temporal characterization of an initial stage of reading in normal subjects and posterior split-brain patients. Brain 2000;123:291-307.

37. Frackowiak RSJ. Human brain function. Elsevier; 2004.

38. Nakamura K, Dehaene S, Jobert A, et al. Task-specific change of unconscious neural priming in the cerebral language network. Proc Natl Acad Sci 2007;104:19643-8.

39. Shaywitz BA, Shaywitz SE, CoDirectors Y. Functional brain imaging in studies of reading and dyslexia. Encycl Lang Lit Dev 2009;12:5-15.

40. Smirni D, Smirni P, Di Martino G, et al. Standardization and validation of a parallel form of the verbal and non-verbal recognition memory test in an Italian population sample. Neurol Sci 2018

41. Smirni D, Smirni P, Di Martino G, Fontana ML, Cipolotti L, Oliveri M, et al. Early detection of memory impairments in older adults: standardization of a short version of the verbal and nonverbal Recognition Memory Test. Neurol Sci 2019;40:97-103.

42. Cavalli E, Colé P, Brèthes H, et al. Ebook reading hinders aspects of longtext comprehension for adults with dyslexia. Ann Dyslexia 2019;69:24359.

43. Reynolds M, Besner D. Reading aloud is not automatic: processing capacity is required to generate a phonological code from print. J Exp Psychol Hum Percept Perform. 2006;32:1303.

44. Stein J. Dyslexia: the role of vision and visual attention. Curr Dev Disord reports 2014;1:267-80.

45. Wolf M, Bowers PG. The double-deficit hypothesis for the developmental dyslexias. J Educ Psychol. 1999; 91:415.

46. Badian NA. Dyslexia and the double deficit hypothesis. Ann Dyslexia 1997;47:69-87.

47. Bowers PG, Wolf M. Theoretical links among naming speed, precise timing mechanisms and orthographic skill in dyslexia. Read Writ 1993;5:69-85.

48. Corcos E, Willows DM. The processing of orthographic information. Vis Process Read Read Disabil 1993;16390.

49. Lovegrove W. Do dyslexics have a visual deficit? In: Studies in Visual Information Processing. Elsevier; 1993. p. 33-49.

50. Willows DM, Corcos E, Kershner JR. Perceptual and Cognitive Factors in Disabled and Normal Readers' Perception and Memory of Unfamiliar Visual Symbols. In: Studies in visual information processing. Elsevier; 1993. 


\section{p. 163-77.}

51. Bishop

DVM language impairment: Same or different? Psychol Bull 2004;130:858.

52. Pennington BF. Using genetics to understand dyslexia. Ann Dyslexia 1989;39:81-93.

53. Thiel A, Schumacher B, Wienhard K, et al. Direct demonstration of transcallosal disinhibition in language networks. Brain 2010;102:610-22.

54. Miles TR, Miles E. Dyslexia: A hundred years on. McGraw-Hill Education (UK); 1999.

55. Snowling MJ. Dyslexia. Blackwell publishing; 2000.

56. Vellutino FR, Fletcher JM, Snowling MJ, Scanlon DM. Specific reading disability (dyslexia): What have we learned in the past four decades? J Child Psychol Psychiatry 2004;45:2-40.

57. Badcock NA, Hogben JH, Fletcher JF. No differential attentional blink in dyslexia after controlling for baseline sensitivity. Vision Res 2008;48:1497502. 